Universidad de Lima

Facultad de Comunicación

Carrera de Comunicación

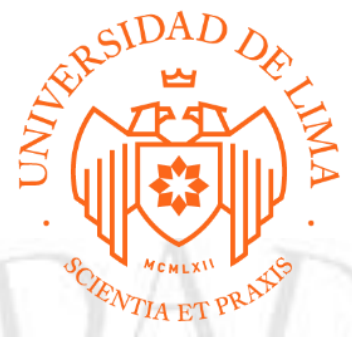

\title{
VIVA LA VIDA
}

Trabajo de Suficiencia Profesional para optar el Título Profesional de Licenciado en Comunicación

\section{Lionel Eduardo Fídel Paredes \\ Código 20111810}

Lima-Perú

14 de mayo de 2019 


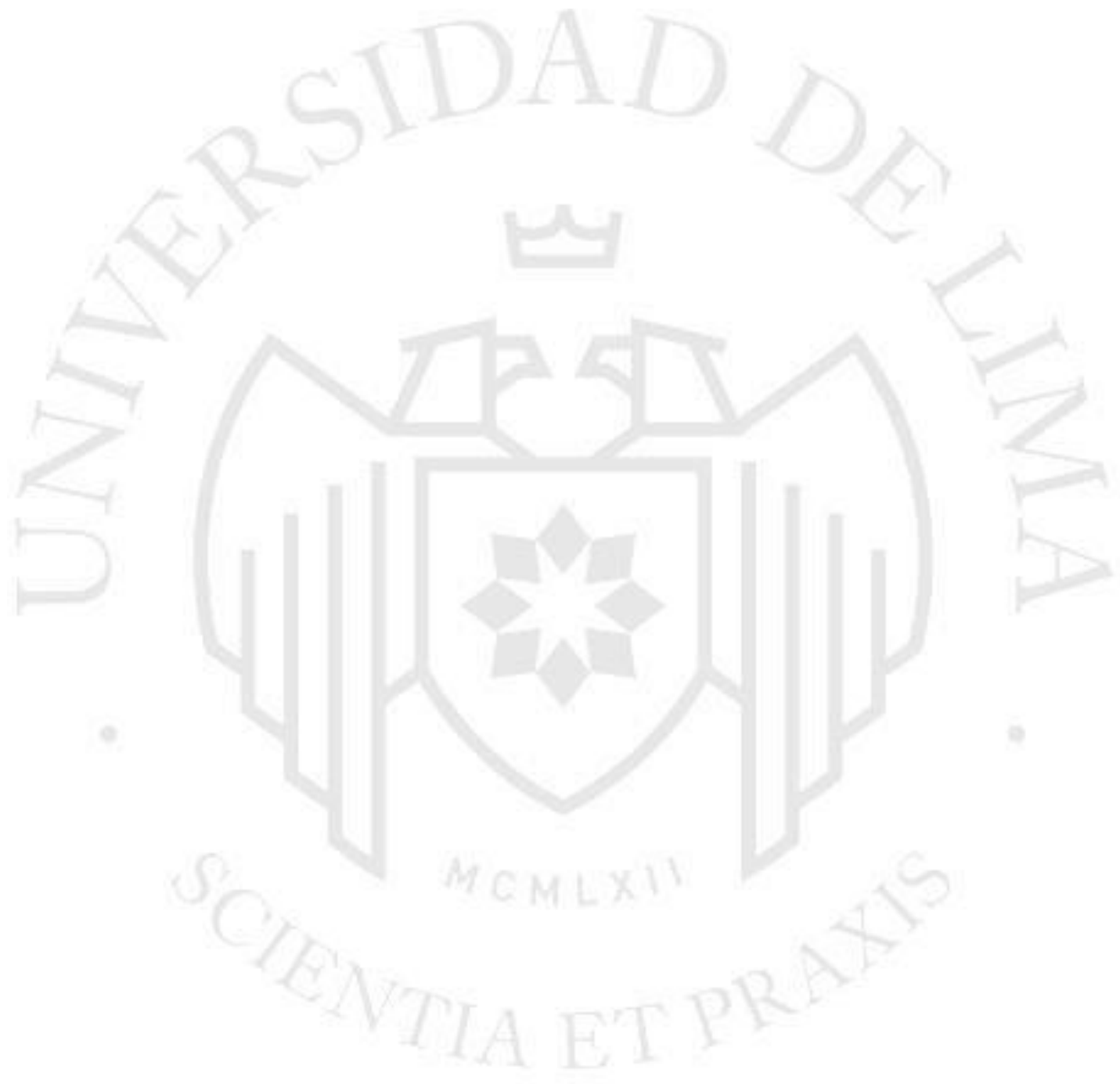




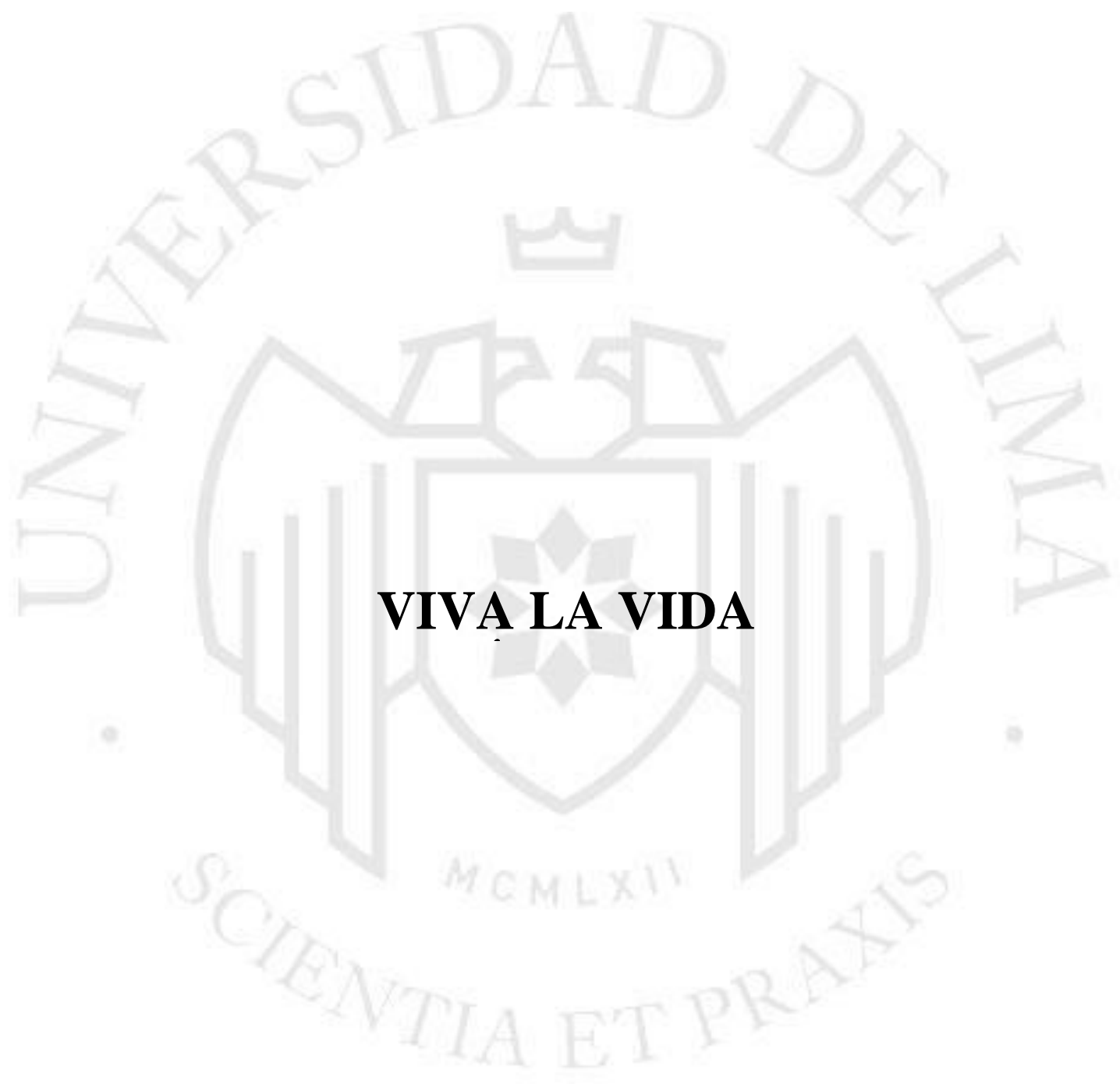




\section{ÍNDICE}

Dirección web de las piezas y producciones de comunicación parte del trabajo p. 5

$\begin{array}{ll}\text { Resumen } & \text { p. } 6\end{array}$

$\begin{array}{ll}\text { 1. Concepto de la serie } & \text { p. } 7\end{array}$

$\begin{array}{ll}\text { 2. Sustentación } & \text { p. } 8\end{array}$

$\begin{array}{ll}2.1 \text { Precedentes o referentes } & \text { p. } 8\end{array}$

2.2 Género, público y tema $\quad$ p. 14

2.3 Ángulo diferencial y posicionamiento $\quad$ p. 15

$\begin{array}{ll}\text { 3. Viva la vida } & \text { p. } 17\end{array}$

\begin{tabular}{l|l}
3.1 Perfil de personajes principales & p. 17
\end{tabular}

$\begin{array}{ll}3.1 .1 \text { Amor } & \text { p. } 17\end{array}$

$\begin{array}{ll}\text { 3.1.2 Marco } & \text { p. } 18\end{array}$

3.2 Sinopsis de episodios p. 19

REFERENCIAS P. 23

ANEXOS P. 25

$\begin{array}{ll}\text { Desglose de producción } & \text { p. } 25\end{array}$

Photoboard p. p. 27

$\begin{array}{ll}\text { Plan de rodaje } & \text { p. } 29\end{array}$

$\begin{array}{ll}\text { Guiones } & \text { p. } 34\end{array}$ 


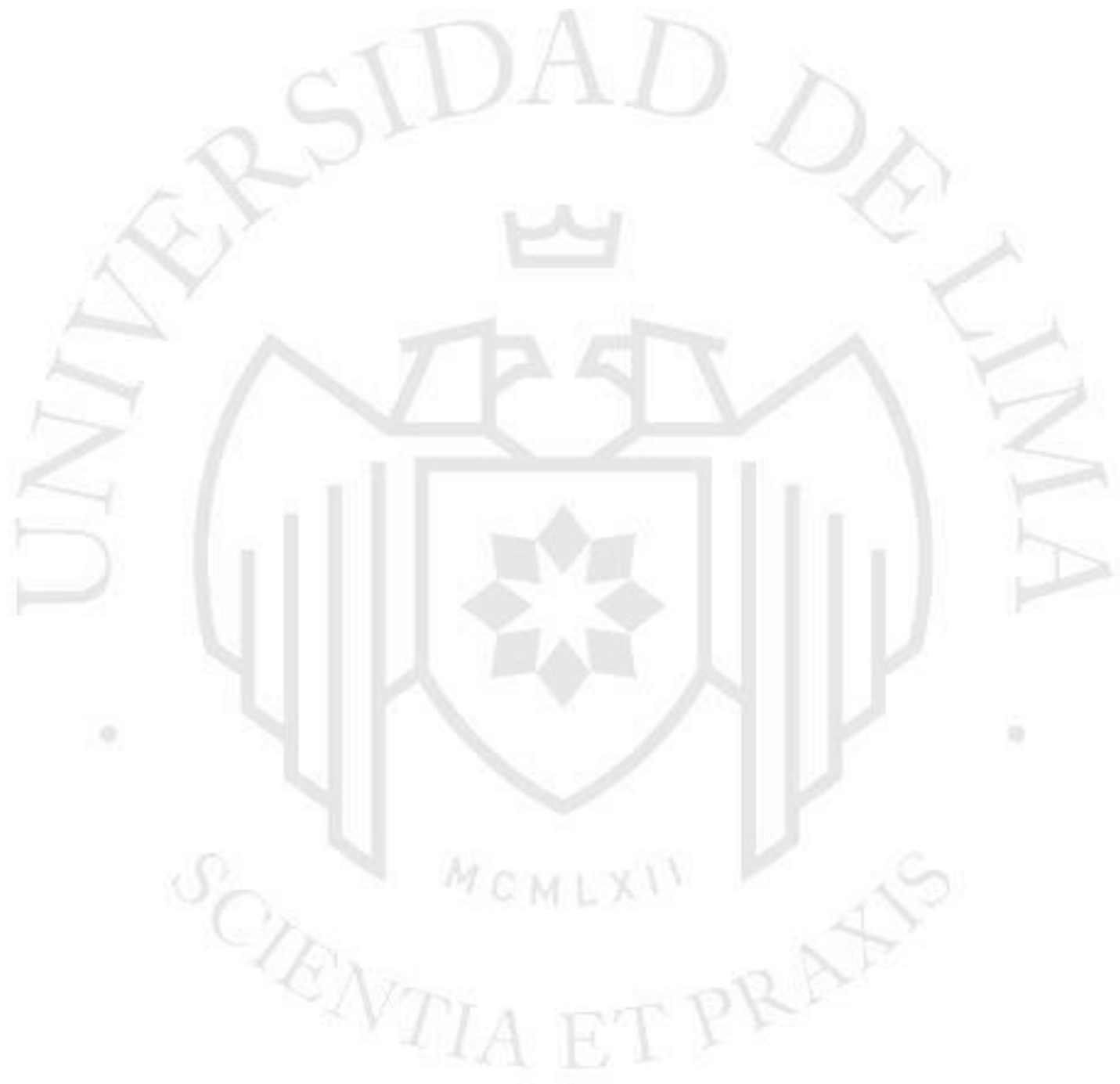


Dirección web de las piezas y producciones de comunicación parte del trabajo

https://drive.google.com/drive/folders/1fVwokH7AreFj2nDIDUN_0M3CFFqeXVTV?u

$\underline{\mathrm{sp}}=$ sharing 


\section{RESUMEN}

Con el nombre Viva la vida se presenta una serie web donde "Marco" el personaje principal se encuentra en una etapa difícil emocionalmente en su vida, sin embargo, todo cambiará cuando un extraño personaje, cuyo nombre es “Amor”, llegue a su vida e intente de todas las maneras posibles hacer que vea la vida con esperanza y amor por medio de la apreciación, entrega y servicio a la vida.

Palabras clave: audiovisual, ficción, amor, esperanza 


\section{Concepto de la serie}

Ángel de miércoles es una serie web donde nuestro personaje principal "Marco" se encuentra en una etapa difícil emocionalmente en su vida, sin embargo, todo cambiará cuando un extraño personaje, cuyo nombre es “Amor”, el cual es su ángel de la guarda, llegue a su vida y este intente de todas las maneras posibles animarlo y que supere esa difícil etapa emocional en su vida por medio de la enseñanza de valores. No obstante, a pesar de que Marco se quiere dar una oportunidad para rehacer su vida y mejorar, las diversas situaciones complicadas y jocosas harán que no sea el camino más fácil para nuestro personaje principal. 


\section{Sustentación}

\subsection{Precedentes o referentes}

"La humanidad vive su presente en medio del consumo rápido, de la amplia y abrumadora oferta: es, en este escenario, en donde se gesta la serie web". (Universidad EAFIT, 2017)

Antes de hablar del género elegido en sí, deseo comentar un poco el caso de un gigante en series: Netflix:

Dentro de la región latinoamericana tenemos a un "gigante de contenido vía streaming", así denomina la BBC a Netflix, en una nota redactada por ellos; el cual llegó en el año 2011. Según un informe elaborado por Sandvine, compañía canadiense especializada en conexiones de banda ancha, para "The Global Internet Phenomena Report", este servicio de streaming se encuentra en los primeros puestos de todos los rankings de datos a nivel global. Y desde ese año este "gigante" fue creciendo, pero no fue hasta el año 2016 donde decidió incrementar ya que el 7 de enero agregó 130 nuevos países a sus catálogos, por otra parte, actualmente se encuentra en 190 países por todo el mundo con un total de 83 millones de suscriptores, una enorme cifra.

Según el artículo: "El Rol de Netflix en el ecosistema de medios y telecomunicaciones: ¿el fin de la televisión y el cine?" "Netflix tiene una importante competencia, no solo en el mercado global sino también en cada país. Solo en Latinoamérica hay más de 90 plataformas, con distintos modelos de negocio". (Siri, 2016) Analizando rápidamente el siguiente cuadro podemos entender a la perfección que, a pesar de tan vasta competencia, el número de abonados de Netflix en Latinoamérica es mucho mayor que el de sus competidores Apple TV, Amazon y Google Play. 
Figura 1. Sitios globales y latinoamericanos de video streaming

\begin{tabular}{|c|c|c|c|}
\hline \multicolumn{2}{|c|}{ Video streaming globales } & \multicolumn{2}{|r|}{ Video streoming latinoamericanos } \\
\hline Operador & Abonados (LATAM) & Pais & Operadores \\
\hline Netflix & 7.300 .000 & Argentina & $\begin{array}{l}\text { Arnet Play, Speedy on Video, Cablevision VOD, } \\
\text { Vesvi, Qubititv, Cinema Argentino, Conectate.gob, } \\
\text { Personal ( } 350.000)\end{array}$ \\
\hline Apple TV & 1.800 .000 & Brasil & $\begin{array}{l}\text { Claro Video, Telecine, Vivo VOD, GVT On Demand, } \\
\text { Muu Globo }\end{array}$ \\
\hline Amazon & & Chile & Claro Video, Movistar Play, Bazuca, VTR VOD \\
\hline \multirow[t]{2}{*}{ Google Play } & 3.100 .000 & Colombia & Claro Video, UNE (NOD), Caracol \\
\hline & & México & $\begin{array}{l}\text { Claro Video, Cablevision VOD, Cinepolis Klic, VEO } \\
\text { (Televisa) }(3.400 .000) \text {, Total Play }\end{array}$ \\
\hline
\end{tabular}

Funtes: Netflix Annual Report, Business Bureau, Egeda (Katv, 2015: 92)

Como dato curioso, después de un análisis de datos publicados por el sitio web Finder.com, podemos decir que los países con mayor contenido disponible son Puerto Rico con 4,880, Trinidad y Tobago con 3565 y República Dominicana con 35523; asimismo estos tres países se encuentran en el top 20 mundial de la misma categoría. A continuación, presento en el Gráfico 1 un cuadro estadístico con el número de películas y programas de TV disponibles en este servidor de streaming de cada país latinoamericano. 


\section{GRÁFICO N¹ \\ Números de películas y programas de TV disponibles en \\ Netflix de cada país de Latinoamérica \\ Febrero \\ 2019}

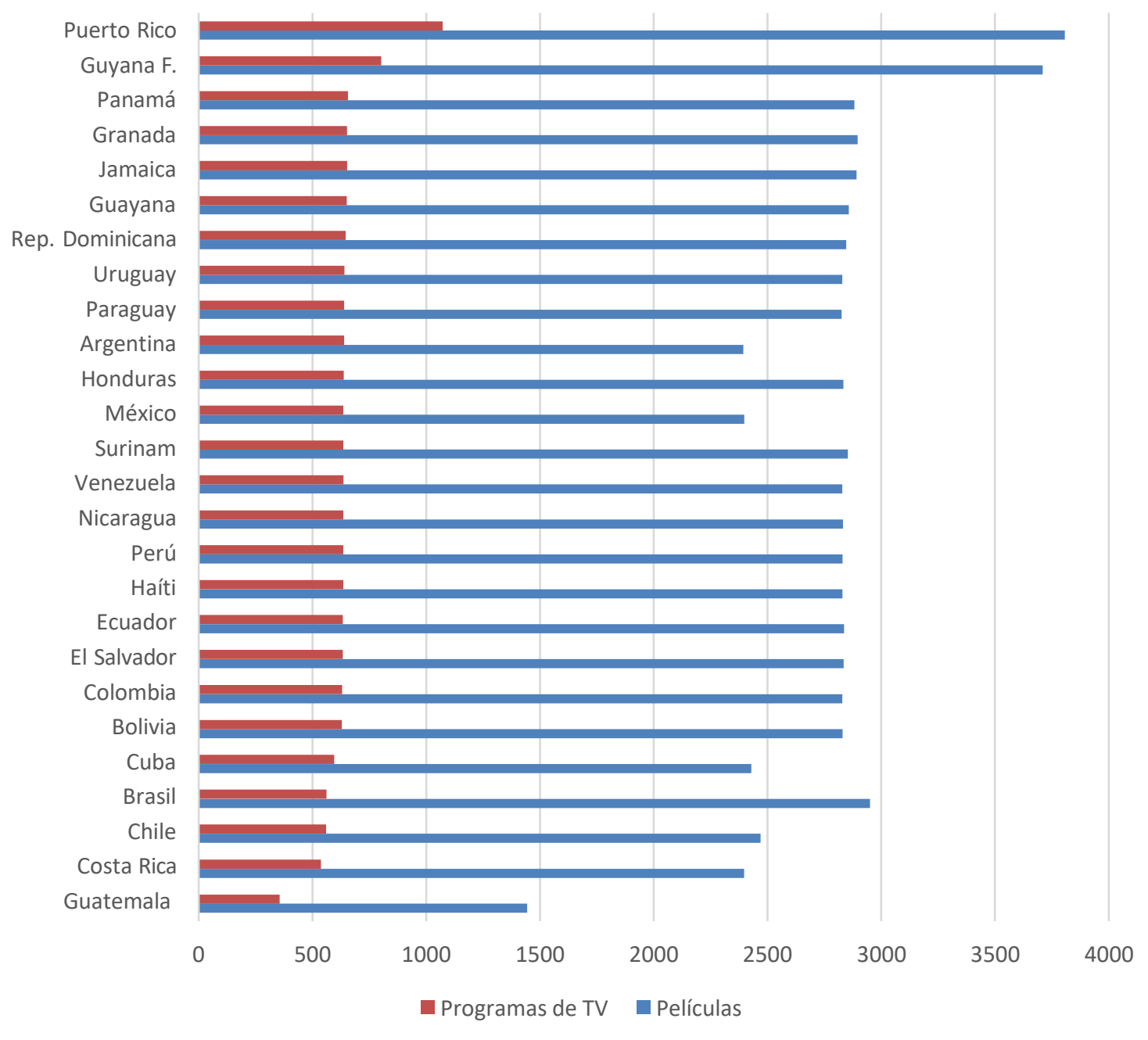

Fuente: www.finder.com/global-netflix-library-totals 
Si bien, el haber explicado en los anteriores párrafos este gran poderío de Netflix nos da un marco y panorama de la calidad del gran consumo de series por streaming que existe por parte de los internautas, no es un referente exacto para la propuesta que se busca plasmar; una serie web con la duración de un minuto, ya que Netflix, dentro de su contenido en series, solo cuenta con productos con un tiempo de duración mucho mayor, teniendo un promedio de cuarenta minutos por capítulo. Además de que Netflix no es una red social.

Por lo mismo, quiero mencionar una de las redes sociales más influyentes en la actualidad y que se encuentre dentro de un crecimiento importante: Instagram, ya que según datos recopilados en diciembre del año pasado en páginas como "Statista" y "Techcrunch" por el diario El Correo Gallego "Instagram ha alcanzado este 2018 los 1.000 millones de usuarios con un tráfico de 500 millones de personas al día entre sus fotos y vídeos. En cuanto a crecimiento por trimestres, supone un $5 \%$, bastante superior al que tienen Facebook o Snapchat, que cuentan con un 3,14\% y 2,13\% respectivamente" (Almeda Omil, 2018). Según el estudio "Digital 2019 Essential Insights into internet, social media, mobile and ecommerce use around the world" es la tercera red social más utilizada por internautas donde "ofrece hasta 58 veces más interacción por seguidor que Facebook y 150 veces más que Twitter, según una encuesta realizada por Forrestser Research, empresa estadounidense de investigación de mercado" (Escobar, 2018), asimismo en Latinoamérica, tenemos a México como el país que posee más usuarios registrados en la región con un total de 16 millones, que es seguido por Argentina, el cual cuenta con 11 millones.

Instagram, habiendo comenzado como una plataforma de fotos, desde hace ya unos años ofreció la opción de poder subir videos con una duración de quince segundos, lo cual abrió una oportunidad muy grande para aquellos que veían más allá de una simple red social para compartir momentos de la vida cotidiana, lo veían como una opción para producir contenido audiovisual de corta duración y que gustara a los usuarios de esta 
plataforma. Por ejemplo: Shieldfive es una miniserie subida el 1 de febrero para esta red social la cual al poco tiempo de vida en internet ya poseía cinco mil seguidores, lo que denota que tuvo una gran acogida por parte del público de instagram, además, actualmente posee más de veintiún mil followers, dato interesante porque dejó de publicar contenido a finales del mismo mes que tuvo su debut; Cambio de Status es otra prueba de que la audiencia ha recibido de buena manera estas series en este formato ya que a los dos meses haber sido estrenada ya contaba con más de cuatro mil seguidores. Por último, una serie con una gran acogida es Víctimas la cual con una semana de estreno y con tres capítulos ya emitidos logró un total de seis mil reproducciones y a lo largo de su trayectoria logro captar la atención de sus receptores llegando a tener en sus 10 primeros capítulos un total de 245 mil reproducciones, incluso logró tener una segunda temporada, hecho que no se ve constantemente en esta plataforma de Instagram en cuestión de series. Esta red social es la más cercana en relación al proyecto que propongo y por esa razón lo tengo como referente principal, por su límite de tiempo que es igual al que sustento yo: series con capítulos de un minuto.

Debido a que esta serie web es sobre comedia he tomado como referente diversas series que han sido exitosas en su momento como:

Kenan \& Kel: Serie emitida por Nickelodeon, trataba las aventuras de un par de amigos que se metían en situaciones y problemas de la manera más hilarante posible. La serie tuvo un rating 7.9. Es una referencia esencial para este proyecto ya que Sentido de Vida tiene a dos personajes como protagonistas que van a pasar por situaciones jocosas como los protagonistas de Kenan \& Kel.

Drake \& Josh: Serie también emitida por Nickelodeon la cual cuenta las aventuras jocosas en diferentes situaciones entre dos hermanastros, consiguió un rating de 7.7. Referente también esencial por la misma razón que Kenan \& Kel ya que es una comedia 
de situación de dos personajes que les pasan cosas graciosas al momento de desarrollar la historia.

Chavo del ocho: Serie mexicana emitida y doblada mundialmente que cuenta las aventuras del personaje de "El chavo" y sus amigos en la famosa vecindad, consiguió un rating de 8.4. Esta serie sirve como referencia no solo por su alto nivel de comicidad, sino también porque a través de la comedia genera valores en cada capítulo que hacen que el espectador pueda aprender estos y pueda aplicarlos en su vida.

Artistically Challenged: Serieweb con una duración de quince segundos donde Nick es un joven artista que pasa por interesantes y jocosas situaciones para sobrevivir en su ciudad. La tomo como referencia por el uso de la comedia en tan poco tiempo ya que saben usar el género en tan solo quince segundos.

EnchufeTV: Serieweb altamente reconocida en youtube que tiene como tema mostrar sketchs en diversas situaciones hilarantes. La manera de jugar con la comicidad al momento de la grabación y la forma de edición que también ayuda mucho a este elemento lo hace un gran referente para este proyecto.

Víctimas: Serieweb chilena con una duración de un minuto del género drama y suspenso que busca captar al espectador desde el primer capítulo donde ya nos muestran de qué va la serie de una manera muy fuerte, tuvo un gran recibimiento en redes sociales en sus dos temporadas. La considero un referente fuerte ya que nos cuentan toda una trama en 1 minuto por capítulo llegando a captar la atención del público y siempre dejándonos con un cliffhanger en cada capítulo que deseas que veas más.

Shieldfive: Serieweb de Inglaterra con una duración de quince segundos que busca enganchar al espectador con cada capítulo, es una de las pioneras desde que Instagram decidió agregar la opción de subir videos a su red social. Un referente bien marcado por 
la manera cómo cuentan una historia en tan poco tiempo y que logra que el espectador quiera consumir cada capítulo con expectativas.

\subsection{Género, público y tema}

Netflix ha ido abarcando cada vez más y más terreno como hemos venido explicando y según la red de blogs Hipertextual: "Netflix se ha especializado en drama y comedia" (Lacort, 2017) con esto no estoy diciendo que Netflix no haya incursionado en otros géneros, seria mentir con esto. Pero queda claro que estos dos géneros son una buena apuesta para este gigante del streaming y que no le ha ido nada mal. Además, no podemos decir que los gustos por estos dos tipos específicos de género se limitan a solo los usuarios que consumen este servicio de streaming ya que los que buscan drama o comedia lo hacen en cualquier tipo de plataforma o medio con el fin de entretenerse, por esta razón tomo como gran referente la audiencia de Netflix, ya que es una muy numerosa y sirve como guía para saber qué géneros son los más consumidos. Gracias a esto he decidido optar por el género de comedia para este proyecto.

El público objetivo y género elegido para esta serie web es el de los adolescentes varones de 12 a 21 años que consuman series cómicas de cualquier nivel socioeconómico de Latinoamérica ya que según una investigación llamada "Diferencias de género en el consumo audiovisual: un experimento de neurociencia sobre spots de televisión" comenta que la comedia ha despertado un mayor interés en el caso de los hombres, y ahondando un poco más a profundidad, que los adolescentes suelen preferir ver programas con contenido de humor y positivo, además de personajes de comedia.

Además, para elegir el horario de publicación de cada capítulo de la serie web hemos visto los resultados de una encuesta realizada por el Instituto Federal de Telecomunicaciones en el año 2017 donde se comenta que "Cerca de las 6 de la mañana, 
es un $34 \%$ más probable que los miembros de Netflix estén viendo comedias en comparación con el resto del día. La gente quiere despertar riendo con personajes como Dev Shah (Master of None), Chandler Bing (Friends) y Charlie Harper (Two and a Half Men), por mencionar algunos". (Reina, 2017), entonces bajo la propuesta que el horario de mañana es de 6:00 a 8:00 a.m. decidimos colocarlo en el horario de 7:00 a.m. teniendo en cuenta que una cantidad adolescentes a partir de las 7:30 a.m. ya asisten al salón de clases, en cambio en el horario propuesto pueden ver nuestra serie cuando estén en camino a su centro de estudios.

Para hablar sobre la temática escogida puedo citar un párrafo del siguiente artículo titulado: "Las preferencias de los adolescentes sobre los personajes televisivos de ficción seriada" donde nos mencionan que "Los adolescentes, en pleno proceso de construcción de su propia identidad, encuentran en los personajes televisivos modelos a través de los cuales ampliar su mundo social y encontrar su lugar en la sociedad" (Korres \& Elexpuru, 2016); bajo esta premisa decidí tocar temas que tengan un contenido de valores morales para estos jóvenes entre los 12 y 21 años ya que los adolescentes al querer forjar una personalidad mi deseo es que puedan tener un buen referente del cual acordarse en el camino de la construcción de su personalidad, lo que nos lleva al ángulo diferencial.

\section{3 Ángulo diferencial y posicionamiento}

Según la revista de cine "Fotogramas" estas 8 series de comedia fueron las mejores en su género en Netflix en el año 2018: Glow, Brooklyn Nine-Nine, Orange is the new black, Paquita Salas, Love, Master of None, Happy! y The Good Place. Si bien es cierto que son series del agrado del público y generen una empatía con los personajes, no hay una entrega de valores directa como lo propone Sentido de vida la cual busca eso como ángulo diferencial, que los adolescentes que la sintonicen puedan recibir los valores que propone la serie como: perseverancia, ayuda al necesitado, valorar a las 
personas entre otros que hará que estos espectadores no solo puedan divertirse aprendiendo de estos valores mediante la comedia, sino puedan llevarlo a su vida y aplicarlo.

De esa manera nos posicionaremos en cuestión de competencia ante las demás series web de comedia como: Artistically Challenged, La nena, De levante, Lavida.es, entre otras que a pesar de tener un cierto nivel de reconocimiento por su contenido y audiencia carecen directamente de valores o la mayoría de sus personajes no son aquellos que los espectadores puedan tomar como ejemplo o referente para su vida. 


\section{3. Ángel de miércoles}

\subsection{Perfil de personajes principales}

\subsubsection{Amor}

Es un ser celeste, un ángel de Dios, el cual lo ha servido durante toda la eternidad. No tiene edad por haber vivido toda su vida, su lugar de nacimiento es el cielo y sus familiares son todos los que habitan con él. Se caracteriza por ser muy obediente a Dios y hacer todo lo que pide con mucha energía y ganas, tiene una personalidad graciosa por lo que sus comentarios suelen tener chistes o comentarios jocosos, por otra parte, nunca ha regresado a la presencia de Dios sin haber completado su tarea, lo que lo hace un ángel muy perseverante.

Posee alas y una aureola en la cabeza característicos de un ángel, y por ser un ser celeste no tiene forma específica, es decir, puede conseguir la forma física que él desee. No utiliza un cierto tipo de vestimenta ya que solo usa una túnica blanca, sin embargo, cuando se hace pasar por un civil en la tierra usa colores vivos que denoten la vitalidad que tiene por ser un ángel.

Su única meta y mayor felicidad en la vida es hacer la voluntad de Dios, y en estos momentos, y desde que Marco nació, es cuidarlo y guiarlo en su vida para que pueda ser feliz e ir al cielo y por esa razón siente un gran cariño y amor hacia él. Amor se considera a sí mismo un fiel obediente a Dios. Su mayor temor es fallarle a Dios, a pesar de que nunca lo ha hecho y Él ha declarado que nunca se sentirá decepcionado de ninguno de sus ángeles, y siente que si le falla no podría volver a presentarse ante Él. 


\subsubsection{Marco}

Su nombre completo es Miguel Marco Gutiérrez Pérez, le gusta que le digan Marco. Tiene 24 años y es de nacionalidad peruana, pertenece al nivel socioeconómico B y su ciudad natal y actual es Lima. Su padre lo abandonó al nacer y su madre siempre trabaja por lo que nunca tuvo una buena atención de niño, aunque ella haya tratado de hacer todo su esfuerzo para que no le falte nada. No tiene hermanos ni contacto con ningún familiar ya que su madre se distanció de su familia por haber tenido un hijo y la echaron de su casa para nunca volver. Es bautizado en la religión católica mas no es un fiel practicante. Se encuentra estudiando la carrera de Ingeniería de Sistemas en la Universidad de Lima encontrándose en el tercio superior cursando el séptimo ciclo pagado por su papá ya que su mamá lo enjuició para que pueda solventarlo hasta que acabará la carrera. Es muy aficionado a todo lo que tenga que ver con la cultura cómic y geek donde descubrió que tenía una capacidad para retener una gran cantidad de información de cualquier tema.

Es de tez mestiza con color de ojos marrón oscuro y cabello negro. Su vestimenta se compone de polos (especialmente de superhéroes), buzos y jeans, todos con colores fríos. No tiene ningún vicio más que quedarse viendo series de netflix, en especial las que tengan que ver con personajes de comic. Físicamente se encuentra bien de salud, sin embargo, emocionalmente no tan bien ya que al no tener una figura paterna de niño y no haber crecido con una buena atención por parte de su madre creció con un sentimiento de soledad por lo que se siente muy solo ya que al final del día siente un gran vació en su casa por no encontrar a nadie. Producto de esa soledad no le ve el sentido a la vida y poco a poco fue volviéndose una persona introvertida y más tímida, esto se vio reflejado en sus relaciones sociales ya que se caracterizaba por ser muy sociable con sus compañeros de clase, además de ser bueno en ajedrez y juegos que impliquen razonamiento, sin embargo, ahora no se comunica mucho con las personas ni con su mejor amigo Diego por una pelea que tuvieron, su rendimiento académico ha bajado considerablemente llegando a pertenecer al tercio inferior llevando un curso en la universidad por segunda 
vez. Su defecto más grande es no tener motivación alguna para con su vida negándose a querer resolver ese problema. Su única meta es sobrevivir a la vida, no realiza nada con pasión como lo hacía antes.

Marco se considera a sí mismo alguien que hace las cosas por cumplir porque sabe que si no las realiza no podrá sobrevivir, sin embargo, no existe una pasión por la cual luchar. No le importa lo que digan los demás porque está ensimismado en su dolor y negación de querer mejorar, además de que no hubo una figura que lo animará e insistiera a mejorar en su vida. Lo que más desea en el mundo es no sentirse solo y se refugia en las películas y videojuegos de superhéroes y cosas geek, aunque ya no lo llenan como antes.

\subsection{Sinopsis de episodios}

Primer Capítulo: No me quiero ir, Señor

\section{Valor: Amor a la vida}

La historia se centra en Marco quien decepcionado de la vida opta por cometer suicidio; sin embargo, es detenido por Amor, su disparatado y distraído ángel guardián, cuya misión será llevarlo por el camino del bien. La situación llega a su fin cuando Marco tropieza de manera fortuita al querer bajarse del techo frente a un desconcertado Amor.

\section{Segundo Capítulo: ¿Ángel de la guarda, dulce compañía?}

\section{Valor: Aceptación}

Luego de la inesperada aparición de Amor y del desafortunado accidente que tuvo Marco, se entabla una conversación entre ambos donde Amor le demuestra que siempre estuvo 
en los momentos más importantes de su vida. El reconocimiento de Amor como ángel guardián da pie a que su amistad comience a forjarse.

\section{Tercer Capítulo: A jalados aprendí}

\section{Valor: Responsabilidad}

Amor, en su faceta de ama de casa, despierta a Marco para que pueda estudiar su curso bica, sin embargo, este preferirá realizar distintas actividades como pasar tiempo con sus figuras de acción y jugar al playstation. Al final Marco se dará cuenta que es mejor jugar cuando sea necesario, cuando la situación lo amerite y no todo el tiempo.

\section{Cuarto Capítulo: Todo por un mostrito}

\section{Valor: Tolerancia}

Marco, cansado de comer menú y cocinar arroz con huevo, intenta darse un gustito yendo a una pollería. Grande es su sorpresa al darse cuenta que está siendo ignorado por todos los mozos, por lo que empieza a perder la paciencia, y es en ese momento cuando Amor lo invita a reflexionar sobre la importancia de respetar los turnos y ser tolerante ante las situaciones adversas.

\section{Quinto Capítulo: ¿Lo guardo para los Guardianes?}

\section{Valor: Honradez}

Asistiendo a un festival de cómics, Marco se da cuenta que no le alcanza el dinero para poder comprar el último set de Los Guardianes de la Galaxia, es entonces cuando se encuentra un sobre lleno de dinero con datos de una persona, así empieza una encrucijada sobre devolver el dinero encontrado o comprar el set que tanto tiempo estuvo esperando; Amor vuelve a influenciar en él. 


\section{Sexto Capítulo: Jaque...ca}

\section{Valor: Humildad}

Marco volverá a participar en un torneo de ajedrez ya que la última vez que lo hizo quedó en 2do lugar; sin embargo, al enterarse de que este año habrán muy pocos competidores no practicará de forma adecuada, situación que Amor presagiará como desfavorable para su protegido. Asimismo, el capítulo presenta a Diego, ex mejor amigo de Marco, quien gana el torneo al realizar una jugada que Marco olvidó por la falta de práctica.

\section{Séptimo Capítulo: Tengo algo que decirte. Edición especial: Marco y Diego}

\section{Valores: Perdón}

Marco no puede creer que Diego le haya vuelto a ganar en todo lo que hace, recuerda las razones por las que se separaron, pero aun así extraña su amistad y los momentos juntos. Amor intentará dar el primer paso por ellos para que de una vez ambos vuelvan a juntarse como en los viejos tiempos, ello con el fin de poder perdonarse y empezar su amistad desde cero.

\section{Octavo Capítulo: Por mi mare}

\section{Gratitud}

Es el cumpleaños de la mamá de Marco y este se siente muy agradecido por el amor y apoyo que le brinda, por lo que quiere darle una sorpresa muy especial realizando una cena, junto a Amor realizan los preparativos, aunque la falta de experiencia en las labores hogareñas hará que la cena sorpresa culmine en caos. Al final junto a su madre limpian la casa, al inicio se siente muy mal, pero se anima al saber que su madre es feliz de tener una familia pequeña pero unida. 


\section{Noveno Capítulo: Estudiando cualquiera sabe}

\section{Valor: Honestidad}

Luego de dar su examen final en la universidad, Marco se da cuenta que el profesor cometió un error y cambió sus resultados con los de Diego, en los cuales daba a Marco la mejor nota del salón y a Diego lo desaprobaba. Amor vuelve a interceder para que confiese la verdad y haga saber del error al profesor. Al final la buena acción sorprende a todos, ya que el profesor cometió otro error al calificar el examen de Marco y este también aprobó el examen.

\section{Décimo capítulo: Mi no tan querido viejo}

\section{Valor: Respeto}

El Día del padre se acerca y Marco comienza a recibir muchos mensajes de su padre, al inicio lo evita, aunque luego de tanta insistencia es convencido por Amor y su madre de reunirse con él. La reunión comienza un poco fría debido a su poca cercanía, pero luego conectan a causa de la confesión del padre sobre la terrible enfermedad que padece, la conversación llega a su punto álgido cuando el padre de Marco confiesa que está tratando de subsanar errores por consejo de su ángel guardián. 


\section{REFERENCIAS}

BBC News Mundo. (05 de Octubre de 2018). BBC. Cómo Netflix está devorando la mayor cantidad de datos en todo el mundo.

Caracol Televisión. (15 de Febrero de 2019). 'De levante. No es amor, es un match', la nueva serie web de Caracol Next.

Domínguez, J. (14 de Octubre de 2018). Fotogramas. LAS MEJORES SERIES DE COMEDIA DE NETFLIX.

Finder. (05 de Febrero de 2019). Netflix International: What movies and TV shows can I watch, and where can I watch them?

Korres, O., \& Elexpuru, I. (2016). Las preferencias de los adolescentes sobre los personajes televisivos de ficción seriada. Trípodos, 20.

Lacort, J. (30 de Mayo de 2017). Hipertextual. Las cifras de Netflix: larga vida al rey. Obtenido de https://hipertextual.com/especiales/las-cifras-de-netflix

Magaña, M. I., \& Marín Díaz, J. P. (20 de Enero de 2016). datasketch. ¿En qué país de Latinoamérica es mejor una noche de 'Netflix \& Chill'?

Redacción Shock. (03 de Diciembre de 2018). Shock. La lección de 'La nena', nueva serie web de Dago García. 
Reina, E. (03 de Junio de 2017). El País. Netflix revoluciona el 'prime time' en América Latina.

Siri, L. (2016). El rol de Netflix en el ecosistema de medios y telecomunicaciones: ¿El fin de la televisión y del cine? Hipertextos, 64.

Tapia Frade, A., Rajas Fernández, M., \& Martín Guerra, E. (2017). GENDER DIFFERENCES IN AUDIOVISUAL CONSUMPTION: A NEUROSCIENCE EXPERIMENT ON TV ADS . Vivat Academia. Revista de Comunicación., 16. 


\section{ANEXOS}

\section{Desglose de producción}

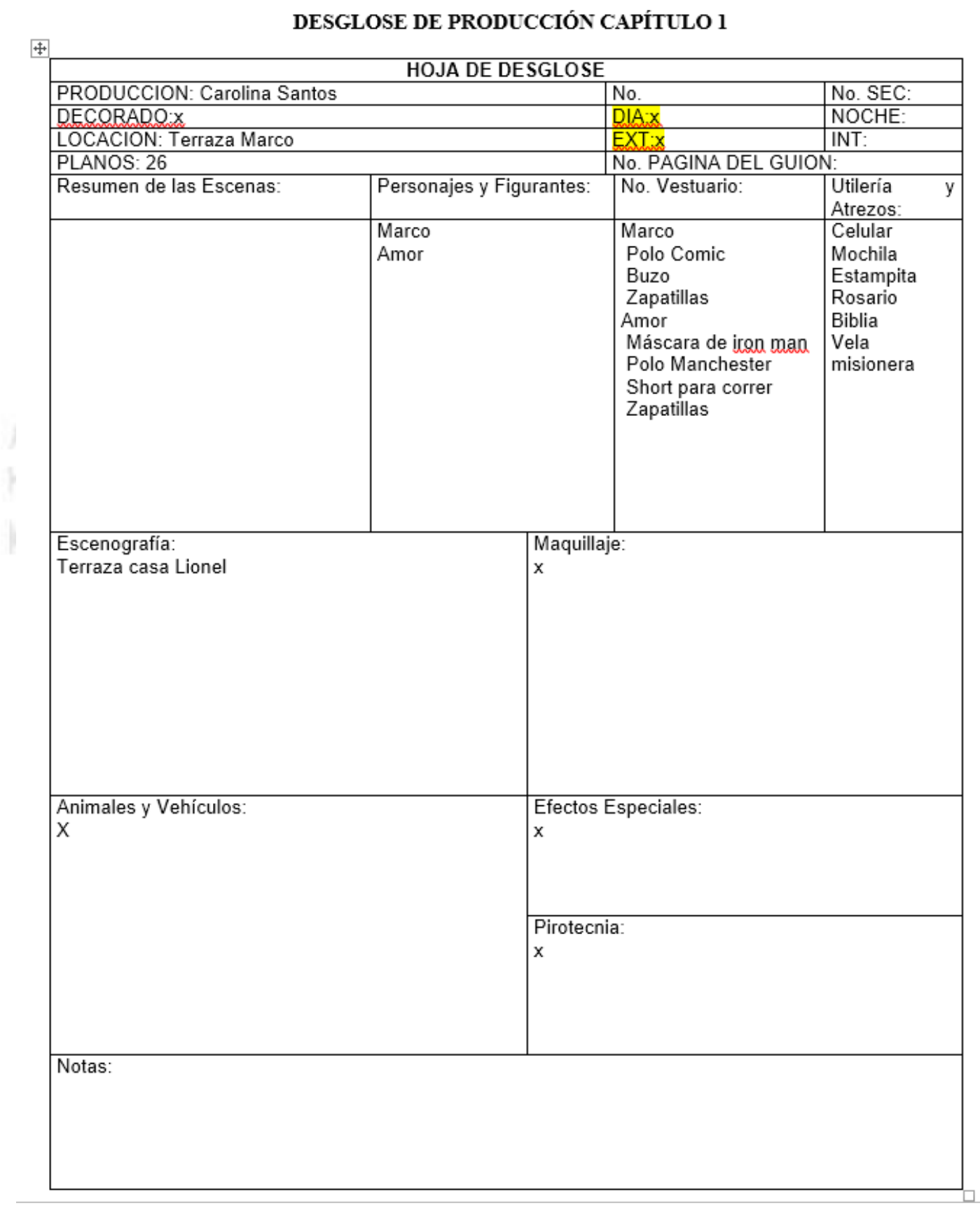


DESGLOSE DE PRODUCCIÓN CAPÍTULO 2

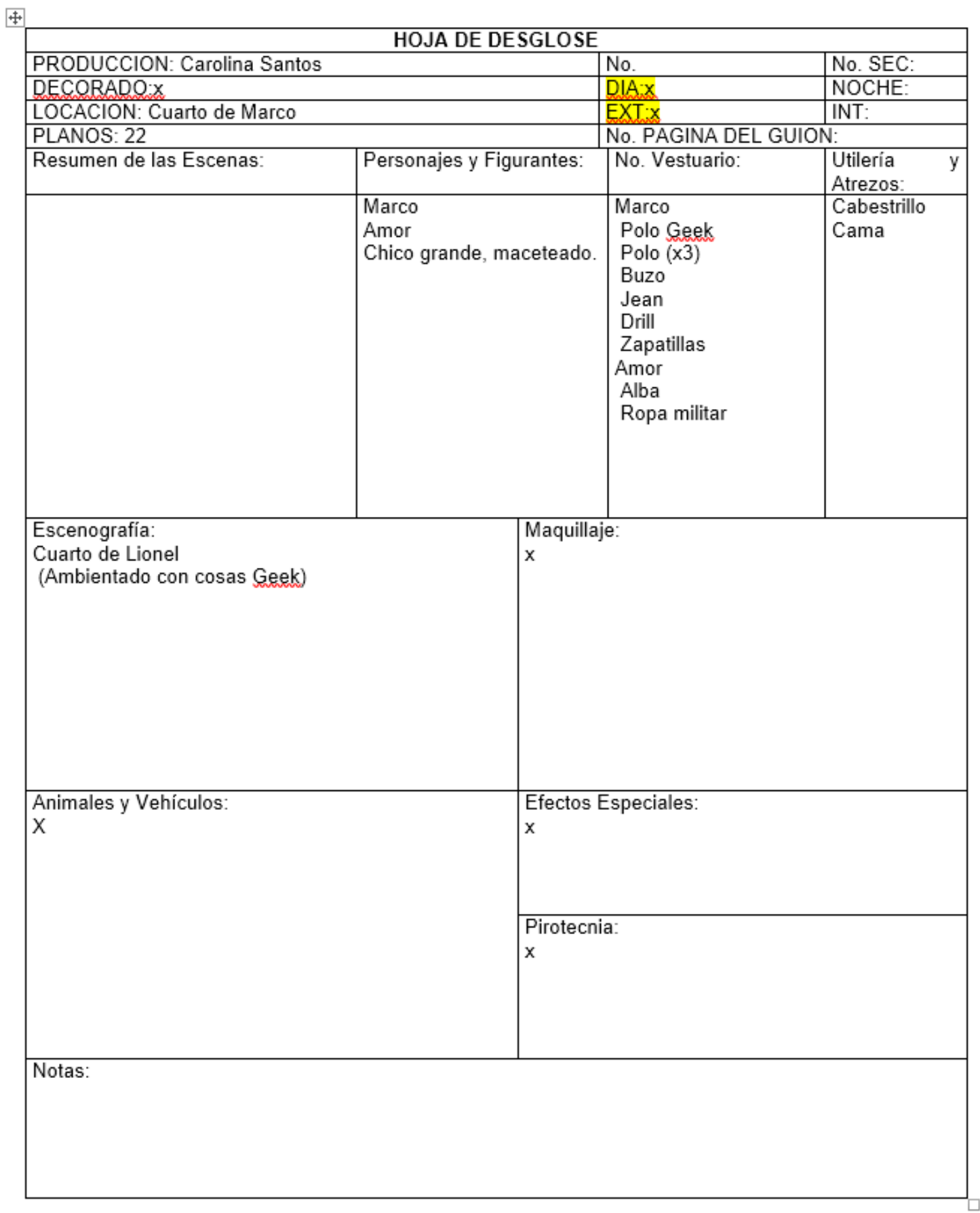


Photoboard

- Capítulo 1

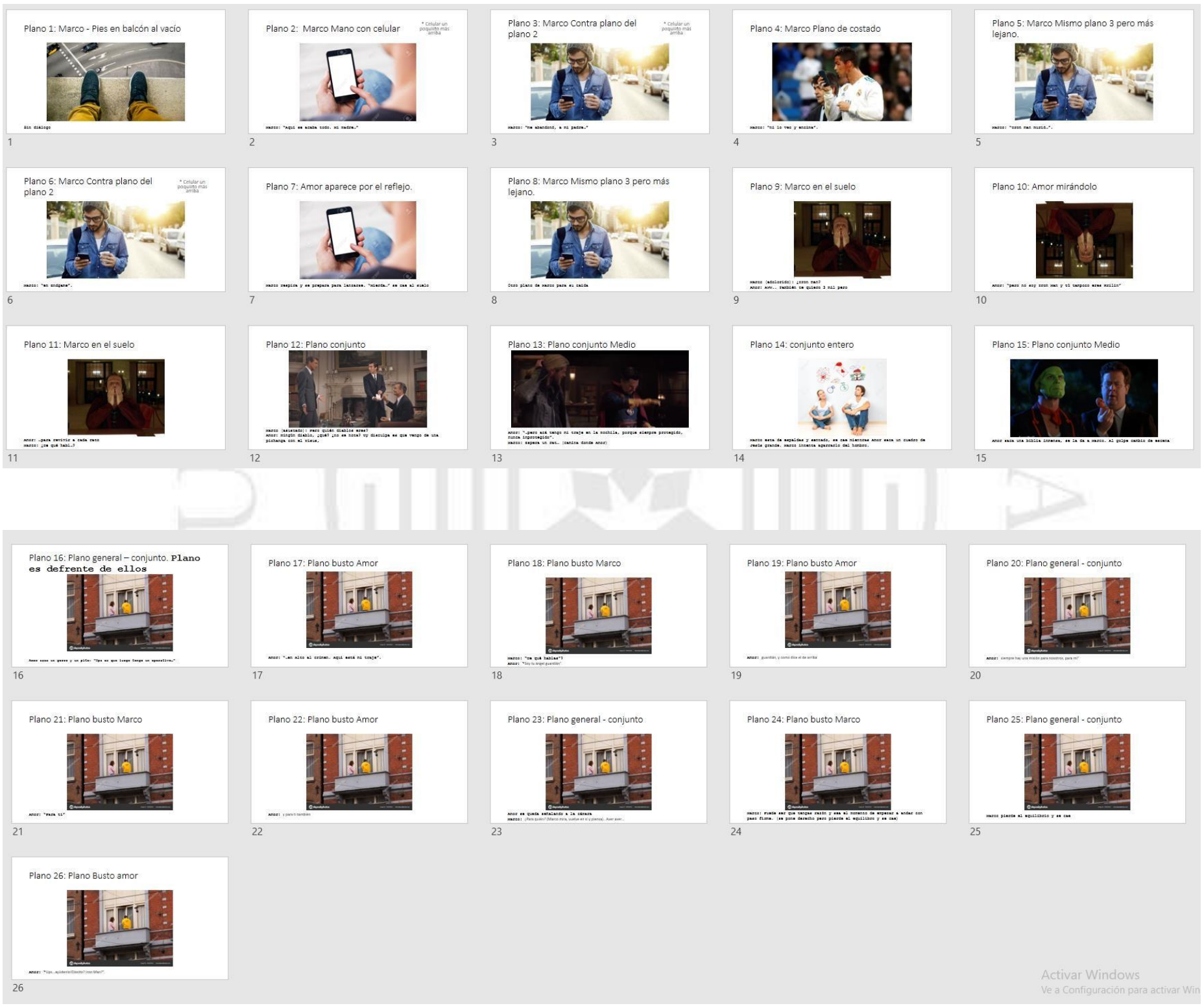


- Capítulo 2

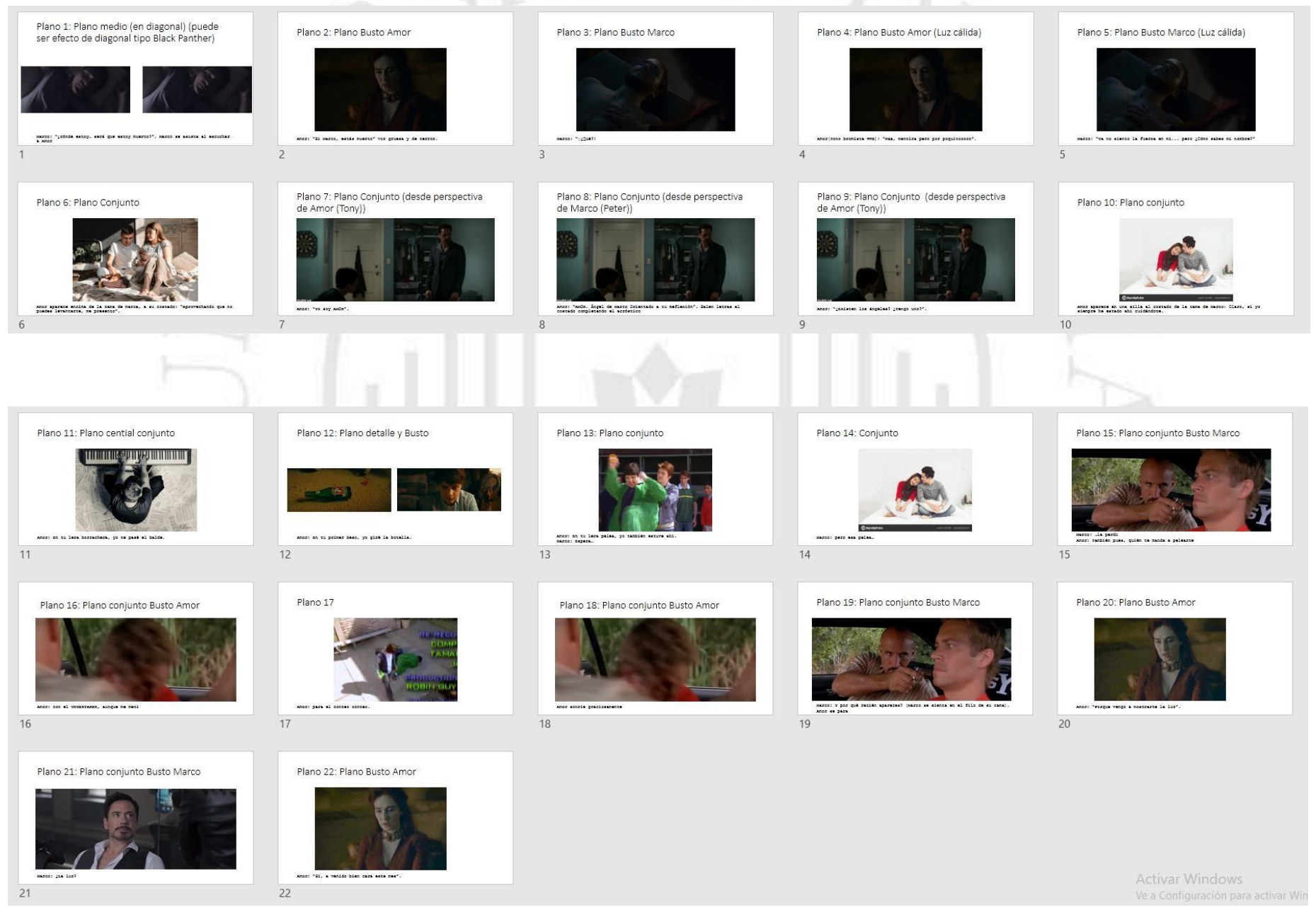




\section{Plan de rodaje}

\begin{tabular}{|c|c|c|c|c|c|c|c|}
\hline Capítulo & HORA & Locación & Escena & Plano & Tipo & Descripción & Nota \\
\hline 2 & 10: $30-10: 45$ & Calle & Extra & 13 & & $\begin{array}{l}\text { Amor: En tu 1era pelea, yo también estuve ahí. } \\
\text { Marco: Espera... }\end{array}$ & \\
\hline 2 & $10: 45-10: 55$ & Calle & Extra & 17 & & Amor: "para el conteo". & \\
\hline$x$ & $10: 55-11: 30$ & \multicolumn{6}{|c|}{ Ensayo } \\
\hline 1 & \multirow[t]{4}{*}{$11: 30-11: 50$} & Terraza & 1 & 16 & Conjunto & $\begin{array}{c}\text { Amor saca un gorro y un pito: "Ups es que luego } \\
\text { tengo un operativo..." }\end{array}$ & \\
\hline 1 & & Terraza & 1 & 20 & Conjunto & $\begin{array}{l}\text { Amor: siempre hay una misión para nosotros, para } \\
\text { mí" }\end{array}$ & \\
\hline 1 & & Terraza & 1 & 23 & Conjunto & $\begin{array}{c}\text { Amor se queda señalando a la cámara } \\
\text { Marco: ¿Para quién? (Marco mira, vuelve en } \\
\text { sí y piensa)... Aver aver... }\end{array}$ & \\
\hline 1 & & Terraza & 1 & 25 & Conjunto & Marco pierde el equilibrio y se cae & \\
\hline 1 & \multirow[t]{4}{*}{$11: 50-12: 10$} & Terraza & 1 & 17 & Busto Amor & Amor: “...en Alto al crimen. Aquí está mi traje”. & \\
\hline 1 & & Terraza & 1 & 19 & Busto Amor & Amor: guardián, y como dice el de arriba & \\
\hline 1 & & Terraza & 1 & 22 & Busto Amor & Amor: y para ti también & \\
\hline 1 & & Terraza & 1 & 26 & Busto Amor & Amor: "Ups...ayúdenlo!Diosito? Iron Man?". & \\
\hline 1 & \multirow{3}{*}{$12: 10-12: 30$} & Terraza & 1 & 18 & Busto Marco & $\begin{array}{l}\text { Marco: “De qué hablas"? } \\
\text { Amor: "Soy tu ángel guardián". }\end{array}$ & \\
\hline 1 & & Terraza & 1 & 21 & Busto Marco & Amor: "Para ti" & \\
\hline 1 & & Terraza & 1 & 24 & Busto Marco & $\begin{array}{l}\text { Marco: Puede ser que tengas razón y sea el } \\
\text { momento de empezar a andar con paso firme. (se } \\
\text { pone derecho pero pierde el equilibro y se cae) }\end{array}$ & \\
\hline
\end{tabular}




\begin{tabular}{|c|c|c|c|c|c|c|c|}
\hline Capítulo & HORA & Locación & Escena & Plano & Tipo & Descripción & Nota \\
\hline 1 & $12: 30-12: 40$ & Terraza & 1 & 2 & Detalle & $\begin{array}{l}\text { Marco con el celular en la mano } \\
\text { Marco: “Aquí se acaba todo. Mi madre..." }\end{array}$ & \\
\hline 1 & & Terraza & 1 & 7 & Detalle & $\begin{array}{c}\text { Amor aparece por el reflejo. } \\
\text { Marco respira y se prepara para lanzarse. "Mierda..." } \\
\text { se cae al suelo }\end{array}$ & \\
\hline 1 & $12: 40-12: 50$ & Terraza & 1 & 3 & $\begin{array}{l}\text { Contra plano del } \\
\text { detalle }\end{array}$ & Marco: "me abandonó, a mi padre..." & \\
\hline 1 & & Terraza & 1 & 6 & $\begin{array}{l}\text { Contra plano del } \\
\text { detalle }\end{array}$ & Marco: "en Endgame". & \\
\hline 1 & $12: 50-13: 00$ & Terraza & 1 & 5 & $\begin{array}{l}\text { Contra plano del } \\
\text { detalle pero más } \\
\text { lejano }\end{array}$ & Marco: "Iron man murió...". & \\
\hline 1 & & Terraza & 1 & 8 & $\begin{array}{l}\text { Contra plano del } \\
\text { detalle pero más } \\
\text { lejano }\end{array}$ & Otro plano de Marco para su caída & \\
\hline 1 & $13: 00-13: 10$ & Terraza & 1 & 4 & Plano perfil medio & Marco: "ni lo veo y encima". & \\
\hline 1 & $13: 10-13: 25$ & Terraza & 1 & 9 y 11 & $\begin{array}{l}\text { Plano frontal Busto } \\
\text { Marco }\end{array}$ & $\begin{array}{c}\text { Marco en el suelo } \\
\text { Marco (adolorido): ¿̇lron man? } \\
\text { Amor: Aww.. También te quiero } 3 \text { mil pero } \\
\text { Amor: ...para revivir a cada rato } \\
\text { Marco: ¿De qué hablas? }\end{array}$ & \\
\hline 1 & & Terraza & 1 & 10 & $\begin{array}{l}\text { Plano frontal Busto } \\
\text { Amor }\end{array}$ & $\begin{array}{l}\text { Amor mirándolo, Amor: "pero no soy Iron Man y tú } \\
\text { tampoco eres Krilin" }\end{array}$ & \\
\hline
\end{tabular}




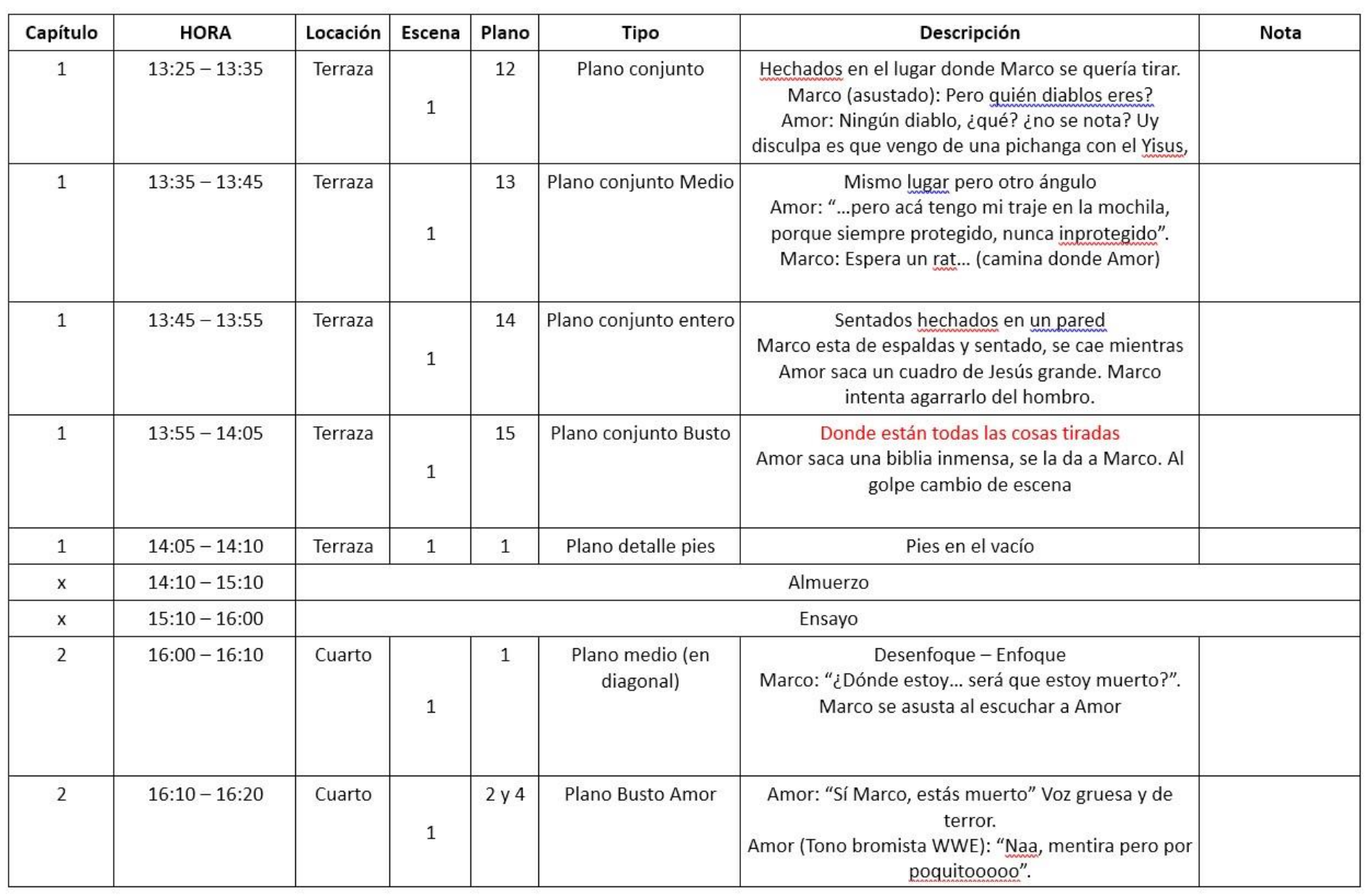




\begin{tabular}{|c|c|c|c|c|c|c|c|}
\hline Capítulo & HORA & Locación & Escena & Plano & Tipo & Descripción & Nota \\
\hline 2 & $16: 20-16: 30$ & Cuarto & 1 & 3 y 5 & Plano Busto Marco & $\begin{array}{c}\text { Marco: “i¿Qué?! } \\
\text { Marco: “Ya no siento la fuerza en mí... pero ¿Cómo } \\
\text { sabes mi nombre?” }\end{array}$ & \\
\hline 2 & $16: 30-16: 40$ & Cuarto & 1 & 6 & Plano Conjunto & $\begin{array}{c}\text { Amor aparece encima de la cama de marca, a su } \\
\text { costado: "Aprovechando que no puedes levantarte, } \\
\text { me presento". }\end{array}$ & \\
\hline 2 & $16: 40-16: 50$ & Cuarto & 1 & 7 y 9 & \begin{tabular}{|c|} 
Plano Conjunto \\
(desde perspectiva de \\
Amor)
\end{tabular} & $\begin{array}{c}\text { Amor: "Yo soy AMOR". } \\
\text { Amor: “¿Existen los ángeles? ¿Tengo uno?". }\end{array}$ & \\
\hline 2 & $16: 50-17: 00$ & Cuarto & 1 & 8 & $\begin{array}{c}\text { Plano Conjunto } \\
\text { (desde perspectiva de } \\
\text { Marco) }\end{array}$ & $\begin{array}{c}\text { Amor: "AMOR. Ángel de Marco Orientado a tu } \\
\text { Reflexión". Salen letras al costado completando el } \\
\text { acróstico }\end{array}$ & \\
\hline 2 & $17: 00-17: 10$ & Cuarto & 1 & $\begin{array}{c}10 y \\
14\end{array}$ & Plano conjunto & $\begin{array}{c}\text { 10: Amor (aparece en una silla al costado de la cama } \\
\text { de Marco): Claro, sí yo siempre he estado ahí } \\
\text { cuidándote. } \\
\text { 14: Marco: pero esa pelea... }\end{array}$ & \\
\hline 2 & $17: 10-17: 20$ & Cuarto & 1 & $\begin{array}{c}16 y \\
18\end{array}$ & $\begin{array}{c}\text { Plano conjunto Busto } \\
\text { Amor }\end{array}$ & $\begin{array}{l}\text { Amor: con el UNDERTAKER, aunque me metí } \\
\text { Amor sonríe graciosamente }\end{array}$ & \\
\hline
\end{tabular}

\begin{tabular}{|c|c|c|c|c|c|c|c|}
\hline Capítulo & HORA & Locación & Escena & Plano & Tipo & Descripción & Nota \\
\hline 2 & $17: 20-17: 30$ & Cuarto & 1 & $\begin{array}{c}15 y \\
19\end{array}$ & $\begin{array}{c}\text { Plano conjunto Busto } \\
\text { Marco }\end{array}$ & $\begin{array}{c}\text { Marco: ...la perdí } \\
\text { Amor: También pues, quién te manda a pelearte } \\
\text { Marco: ¿Y por qué recién apareces? (Marco se sienta } \\
\text { en el filo de su cama). } \\
\text { Amor se para }\end{array}$ & \\
\hline 2 & $17: 30-17: 40$ & Cuarto & 1 & $\begin{array}{c}20 y \\
22\end{array}$ & Plano Busto Amor & $\begin{array}{l}\text { Amor: "Porque vengo a mostrarte la luz". } \\
\text { Amor: "Sí, a venido bien cara este mes". }\end{array}$ & \\
\hline 2 & $17: 40-17: 50$ & Cuarto & 1 & 21 & Plano Busto Marco & Marco: ¿La luz? & \\
\hline 2 & $17: 50-18: 00$ & $\begin{array}{l}\text { Lavadero } \\
\text { tercer } \\
\text { piso }\end{array}$ & Extra & 11 & Plano cential conjunto & Amor: En tu 1era borrachera, yo te pasé el balde. & \\
\hline 2 & $18: 00-18: 10$ & $\begin{array}{l}\text { Mezanine } \\
\text { 3er piso }\end{array}$ & Extra & 12 & $\begin{array}{c}\text { Plano detalle (botella) } \\
\text { y Busto (marco) }\end{array}$ & Amor: En tu primer beso, yo giré la botella. & \\
\hline & & & & & & & \\
\hline
\end{tabular}




\section{Guiones}

\section{Primer capítulo: No me quiero ir, Señor}

\section{(EXT. DÍA BALCÓN/ CASA DE MARCO)}

Se observa unos pies al filo de un balcón de una casa de4 pisos, se enciende la cámara frontal de un celular

\section{MARCO}

(devastado grabando en modo selfie su despedida)

Aquí se acaba todo. Mi madre me abandonó, a mi padre ni lo veo, y encima Iron Man murió en Endgame.

Amor aparece usando una máscara de Iron Man, saludándolopor el reflejo de la cámara en modo selfie

\section{MARCO}

(Gritando) Mierda, Iron Man?

Marco logra recuperar su equilibrio

\section{AMOR}

(Risueño quitándose la máscara De Iron Man)

También te quiero 3 millones, pero no soy Iron Man y tú tampoco eres Krillin para querer revivir a cada rato

Suena música de Dragon Ball

\section{MARCO}

(Conmosionado quitándose la pelada de jebe)

¿De qué hablas? ¿Pero quién diablos eres tú?

\section{AMOR}

\section{(hilarante vestido como futbolista)}

Ningún diablo,¿qué? ¿no se nota? uy disculpa es que vengo de un relámpago en el cielo, pero acá tengo mi traje en la mochila, porque siempre protegido, nunca inprotegido.

Amor saca 3 objetos de su mochila de forma sucesiva. 1. estampita o un rosario 2. una vela misionera o una bibliay un gorro y pito de guachiman

\section{(CONTINÚA)}




\section{CONTINÚA:}

2.

\section{AMOR}

Ups es que luego tengo un operativo en Alto al crimen. Aquí está mi traje

AMOR

(Sonriendo) Ya ves, acá está

\section{MARCO}

¿De qué hablas?

\section{AMOR}

(relajado y rompe la cuarta pared al final de su oración dirigiéndose al público) Soy tu ángel guardián, y como dice el de arriba siempre hay una misión para nosotros, para mí, para ti y para ti también

$$
\text { mira y toca el lente de la cámara }
$$

\section{MARCO}

¿Para quién?

\section{Reflexiona unos segundos}

\section{MARCO}

Puede ser que tengas razón y seael momento de empezar a andar conpaso firme.

intenta retroceder ycae

\section{AMOR}

(extrañado buscando ayuda) Ups...ayúdenlo!Diosito? Iron Man? 


\section{Segundo capítulo: ¿Ángel de la guarda, dulce compañía?}

\section{INT.CUARTO DE MARCO}

Marco despertando en su cama usando un cabestrillo

$$
\begin{gathered}
\text { MARCO } \\
\text { DÓNDE ESTOY? SERÁ QUE ESTOY MUERTO? }
\end{gathered}
$$

Amor aparece desde abajo de la cama vestido de ángel

$$
\text { AMOR }
$$

Sí, Marco, estás muerto

\section{MARCO}

¡qué!

\section{AMOR}

(Tono bromista WWE)

Naa, mentira pero por poquitooooo Marco intenta levantarse, pero no puede

\section{MARCO}

(Desorbitado yhaciendo referencia a Star War)

Ya no siento la fuerza enmí... pero ¿Cómo sabes mi nombre?

Amor camina paso ligero comomilitar

\section{AMOR}

(Estilo pretensioso) Aprovechando que no puedes levantarte,me presento. Yo soy

AMOR Ángel de Marco Orientado a tu Reflexión

\section{MARCO}

(Incrédulo)

¿Existen los ángeles? ¿Tengo uno?

\section{AMOR}

(Emocionado pálmandose el pecho)

Claro, sí yo siempre he estado ahí cuidándote. En tu 1era borrachera, yo te pasé el balde.

En tu primer beso, yo giré la botella. En tu 1era pelea, yo también estuve ahí.

(CONTINUED)

\section{CONTINUED:}


MARCO

(Dudoso)

Oye,pero esa pelea la perdí

AMOR

(alegre)

También pues, quién te manda a pelearte con el UNDERTAKER, aunque me metí... para el conteo conteo.

MARCO

Y por qué recién apareces?

AMOR

Porque vengo a mostrarte la luz

MARCO

¿La luz?

Sacando un recibo deluz

AMOR

(extrañado)

Sí, ha venido bien cara este mes 


\title{
Tercer capítulo:
}

\section{A jalados aprendí}

\section{INT.DORMITORIO DE MARCO}

Amor vestido como ama de casa limpia el cuarto de Marco,lo despierta y sacude con el plumero.

\section{AMOR}

Uy acá también está cochino, no mejor que se bañe. Marco levántate que tienes que ir a estudiar

\section{MARCO}

Déjame dormir, hoy no tengo clase

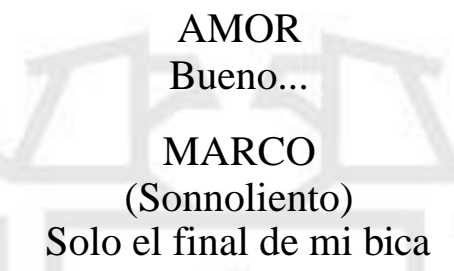

AMOR

¡Qué! anda estudia, no puedes volver a jalar mate básica. Tu amigo con el que ingresaste ya es jp y tu eres su alumno

Se levanta de su cama por el comentario

\section{MARCO}

(Indignado)

Tienes razón estoy atrasado, esta vez sí apruebo

\section{INT.ESCRITORIO DE MARCO}

\section{MARCO SENTÁNDOSE EN SU ESCRITORIO, LLENO DE LIBROS.}

Posteriormente, saca unos juguetes

\author{
MARCO \\ (filósofo)
}

Ahora sí sabré el significado de la fuerza

AMOR

(limpiando de lejos)

Qué buen muchacho, cómo estudia 
(CONTINÚA)

CONTINÚA:

MARCO

(entusiasmado con sus juguetes)

Fuerza es aquello que le brinda poder al jedi

\section{AMOR}

Estás estudiando no?

Lanza los juguetes

MARCO

Sí, sí estoy en eso

\section{INT. SALA DE MARCO}

Marco se encuentra jugando playstation en susillón, mientras Amor está cocinando en otra habitación

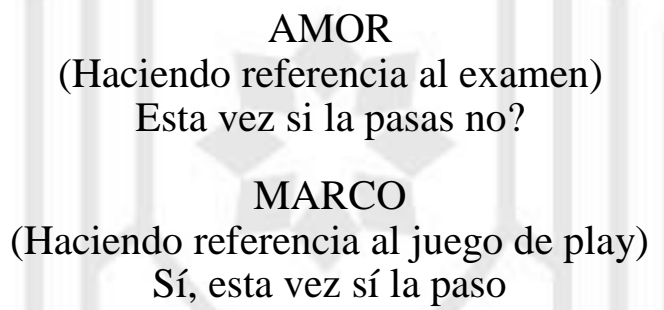

INT. SALA

Marco entra triste a la sala, mientras que Amor loespera sentado jugando play

AMOR

¿Qué tal el examen?

\section{MARCO}

Mal, ya me fui a la trica, debí haber estudiado más cuando pudey tontear después

\section{AMOR}

Sí, para la próxima estudia y no flojees, Lo positivo de esto es que te seguirá enseñando tu compañero con el que ingresaste... un partidito? 